Proyecciones Journal of Mathematics

Vol. 36, $\mathrm{N}^{o}$ 1, pp. 131-148, March 2017.

Universidad Católica del Norte

Antofagasta - Chile

\title{
Representation fields for orders of small rank
}

\author{
Luis Arenas-Carmona * \\ Universidad de Chile, Chile \\ Received : September 2016. Accepted : November 2016
}

\begin{abstract}
A representation field for a non-maximal order $\mathcal{H}$ in a central simple algebra is a subfield of the spinor class field of maximal orders which determines the set of spinor genera of maximal orders representing $\mathcal{H}$. In our previous work we have proved the existence of the representation field for several important families of suborders, like commutative orders, while we have also found examples where the representation field fails to exist. To be precise, we have found full-rank orders, in central simple algebras of dimension 9 or larger over a suitable field, whose representation field is undefined. In this article, we prove that the representation field is defined for any order $\mathcal{H}$ of rank $r \leq 7$. This is done by defining representation fields for arbitrary representations of orders into central simple algebra and showing that the computation of these generalized representation fields can be reduced to the case of irreducible representations. The same technique yields the existence of representation fields for any order in an algebra whose semi-simple reduction is commutative. We also construct a rank-8 order, in a 16-dimensional matrix algebra, whose representation field is not defined.
\end{abstract}

Keywords : central simple algebras, maximal orders, representations of orders, spinor genera.

Subjclass : 11S45-11R52-11R37-16G30.

*Supported by Fondecyt, grant no.1140533. 


\section{Introduction}

Let $K$ be a global field. Let $\Theta$ be a central simple $K$-algebra ( $K$-CSA or CSA when $K$ is clear from the context). Let $\mathcal{O}=\mathcal{O}_{K, S}$ be the ring of $S$ integers in $K$, for some finite set $S$ of places in $K$ containing the archimedean places if any. Finally, let $\Sigma$ be the spinor class field for the set $\Omega$ of maximal $S$-orders in $\Theta$ as defined in [3], i.e., $\Sigma / K$ is an abelian extension that classifies maximal orders of $\Theta$ into spinor genera, in the sense that there exists an explicit map

$$
\rho: \Omega \times \Omega \rightarrow \operatorname{Gal}(\Sigma / K)
$$

with the following properties:

1. $\mathcal{D}$ and $\mathcal{D}^{\prime}$ are in the same spinor genus if and only if $\rho\left(\mathcal{D}, \mathcal{D}^{\prime}\right)=I d_{\Sigma}$, and

2. $\rho\left(\mathcal{D}, \mathcal{D}^{\prime \prime}\right)=\rho\left(\mathcal{D}, \mathcal{D}^{\prime}\right) \rho\left(\mathcal{D}^{\prime}, \mathcal{D}^{\prime \prime}\right)$, for any triple $\left(\mathcal{D}, \mathcal{D}^{\prime}, \mathcal{D}^{\prime \prime}\right) \in \Omega^{3}$,

$[4, \S 1]$. The importance of this concept lies in the fact that spinor genera and conjugacy classes coincide whenever the group $\Theta^{*}$ has strong aproximation with respect to the set $S$. This happens in particular, when $S$ is the set of archimedean places on a number field $K$ and $\Theta_{\wp}$ is not the real quaternion division algebra for at least one such place, e.g., when the dimension of $\Theta$ is larger than 4. In this case, the spinor class field gives much information on the set $\Theta^{*} \backslash \Omega$ of conjugacy classes of maximal $S$-orders, e.g., the number of such conjugacy classes is $\left|\Theta^{*} \backslash \Omega\right|=[\Sigma: K]$. The set of spinor genera of maximal orders also plays an important role, when $K$ is a global function field, in the description of a fundamental set for the action, of certain arithmethically interesting subgroups of the projective general linear group $P G L_{2}(K)$, on some local Bruhat-Tit trees [5]. The corresponding quotient graphs are closely related to the function field analog of Shimura curves [13]. The extension of this theory to number fields also encodes important arithmetic information, for example in [7] we characterize, in a definite quaternion algebra, the orders of maximal rank containing cubic roots of unity, in terms of the corresponding quotient graphs.

Describing the set of orders in a genus containing a copy of a given suborder is known as the selectivity problem, and its understanding is critical for the construction of isospectral but non-isometric Riemannian manifolds. In the indefinite case, for example for algebras of dimension 9 or larger over number fields, this problem can be solved by the theory of 
spinor genera alone. For any suborder $\mathcal{H}$ there exists a lower representation field $F=F_{-}(\mathcal{H})$, which is the largest subfield satisfying

$$
\left.\mathcal{H} \subseteq \mathcal{D} \cap \mathcal{D}^{\prime} \Rightarrow \rho\left(\mathcal{D}, \mathcal{D}^{\prime}\right)\right|_{F}=I d_{F}, \quad \forall\left(\mathcal{D}, \mathcal{D}^{\prime}\right) \in \Omega^{2} .
$$

In [4] we gave an explicit description of the field $F$ that is valid for an arbitrary order in $\Theta$. There exists also an upper representation field $F^{\prime}=F^{-}(\mathcal{H})$, which is the smallest field satisfying the converse property: If $\mathcal{H} \subseteq \mathcal{D}$ and $\left.\rho\left(\mathcal{D}, \mathcal{D}^{\prime}\right)\right|_{F^{\prime}}=I d_{F^{\prime}}$, then $\mathcal{H}$ is contained in some order in the spinor genus of $\mathcal{D}^{\prime}$. When $F=F^{\prime}$, we call it the representation field $F(\mathcal{H})$ of $\mathcal{H}$ and say that the representation field for $\mathcal{H}$ is defined (or exists). In this case the number of spinor genera representing $\mathcal{H}$ is $[\Sigma: F]$ and the question of which spinor genera have a representative containing $\mathcal{H}$ (or equivalently, a conjugate of $\mathcal{H}$ ) can be completely solved in terms of the distance $\rho$ mentioned above. In fact, if $\mathcal{D} \in \Omega$ is an order containing $\mathcal{H}$, a spinor genera $\operatorname{Spin}\left(\mathcal{D}^{\prime}\right) \subseteq \Omega$ has a representative containing $\mathcal{H}$ if and only if $\rho\left(\mathcal{D}, \mathcal{D}^{\prime}\right)$ is trivial on $F$. In particular, if the group $\Theta^{*}$ has strong approximation and $F(\mathcal{H})=K$, every maximal order represents $\mathcal{H}$.

The existence of the representation field has been proved for several important families of orders. In [3], we proved the existence of the representation field whenever $\mathcal{H}$ is commutative, extending previous results of Chevalley [9], Chinburg and Friedman [10], or Linowitz and Shemanske [12]. Later we proved that when $\Theta$ is a division algebra, the representation field of a commutative suborder can be at most a quadratic extension $L$ of $K[6]$. Here we prove the following existential result for orders of small rank:

Theorem 1 Let $K$ be a global field, let $\Theta$ be a $K-C S A$, and let $S$ be a non-empty finite set of places in $K$ containing the archimedean places if any. Then the representation field $F(\mathcal{H})$ is defined for any $S$-order $\mathcal{H} \subseteq \Theta$ whose rank does not exceed 7.

The main tool in our proof of Theorem 1 is an extension of the notion of representation field to representations, rather than suborders, so that computation of the spinor image (as defined in $\S 2$ ) can be reduced to irreducible representations. The same technique yields the following generalization of the main result in [3]:

Theorem 2 Let $K$ be a global field, let $\Theta$ be a $K-C S A$, and let $S$ be 
a non-empty finite set of places in $K$ containing the archimedean places if any. Let $\mathcal{H} \subseteq \Theta$ be an $S$-order. If the maximal semisimple quotient of the algebra $L=K \mathcal{H}$ is commutative, then the representation field $F(\mathcal{H})$ is defined.

On the other hand, in [2] we found a rank-9 order in a 9-dimensional CSA for which the representation field is not defined. Here we prove that the bound 7 in Theorem 1 is optimal by providing a counterexample of rank 8. In all that follows we let $\operatorname{Mat}_{n}(E)$ denote the algebra of $n$ by $n$ matrices over the field $E$.

Theorem 3 Let $K$ be a number field whose ideal class group has an element of order 4 . Then there exists a quadratic extension $F / K$ and an order $\mathcal{H}$ of maximal rank in $L=\operatorname{Mat}_{2}(F) \subseteq M_{4} t_{4}(K)$ for which the representation field is not defined.

Note that $\operatorname{Mat}_{2}(F)$ is identified with a sub-ring of $\operatorname{Mat}_{4}(K)$ via the natural representation of $M_{a t}(F)$ on $F^{2} \cong K^{4}$. As far as we know, this is the first example of an order of non-maximal rank whose representation field fails to exist.

\section{Representation fields for representations}

Let $K$ be a field, and let $B$ be a finite dimensional central division $K$ algebra. Let $L$ be an arbitrary finite dimensional $K$-algebra. By a $B$ representation of $L$ we mean a $K$-algebra homomorphism $\phi: L \rightarrow \operatorname{Mat}_{n}(B)$. For any such $\phi$, the abelian group $B^{n}=B \times \cdots \times B$, regarded as the space of column vectors, has a natural $(L, B)$-bimodule structure given by the products $l \cdot v * b=\phi(l) v b$, for any $(l, v, b) \in L \times B^{n} \times B$. We recall a few well known facts from representation theory:

- A $B$-subspace $W \subseteq B^{n}$ is $L$-invariant if and only if it is an $(L, B)$ sub-bimodule.

- For any chain of $(L, B)$-sub-bimodules $\{0\}=M_{0} \subseteq M_{1} \subseteq \cdots \subseteq$ $M_{r}=B^{n}$ there exists a $B$-basis $T$ of $B^{n}$, such that the matrix of $\phi(l)$, for any element $l \in L$, with respect to the basis $T$, has a block 
decomposition of the form:

$$
\left(\begin{array}{cccc}
a_{11} & a_{12} & \cdots & a_{1 r} \\
0 & a_{22} & \cdots & a_{2 r} \\
\vdots & \vdots & \ddots & \vdots \\
0 & 0 & \cdots & a_{r r}
\end{array}\right)
$$

where each $a_{i j}=a_{i j}(l)$ is a block with $\operatorname{dim}_{B}\left(M_{i}\right)$ rows and $\operatorname{dim}_{B}\left(M_{j}\right)$ columns. The map $l \mapsto a_{i i}(l)$ is the representation corresponding to the bi-module $\tilde{M}_{i}=M_{i} / M_{i-1}$.

- If $M=B^{n}$ has no non-trivial proper sub-bimodules, or equivalently, if the representation $\phi$ is irreducible, then $\phi(L)$ is a simple algebra.

- Given two representations, $\phi, \psi: L \rightarrow \operatorname{Mat}_{n}(B)$, there exists $a \in$ $\operatorname{Mat}_{n}(B)^{*}$ satisfying $\psi(l)=a \phi(l) a^{-1}$ for every element $l$ in $L$, if and only if the bimodules defined by $\phi$ and $\psi$ are isomorphic.

In the remaining of this section $K, \Theta, \mathcal{O}$ are as in the introduction. Let $\Pi(K)$ be the set of all places, both archimedean and non-archimedean, in $K$. Let $S \subseteq \Pi(K)$ be a finite nonempty set, containing the archimedean places, if any, and let $U=\Pi(K)-S$. We refer to $U$ and $S$, respectively, as the set of finite and infinite places of $K$. Every definition that follows can be extended to the projective case, where $K$ is a global function field, $S=\emptyset$, while lattices and orders can be interpreted in a sheaf-theoretical context (see the remark at the end of this section). For every place $\wp$ we let $I^{\wp}$ be the maximal ideal corresponding to $\wp$. Note that $m_{\wp}=I_{\wp}^{\wp}$ is the maximal ideal of the complete local ring $\mathcal{O}_{\wp} \subseteq K_{\wp}$.

In all that follows, $\mathcal{H}$ is an $S$-order on a finite dimensional $K$-algebra $L$, i.e., a lattice $\mathcal{H} \subseteq L$ that is also a subring of $L$ and satisfies $K \mathcal{H}=L$. Let $L_{\mathcal{A}}$ be the adelization of $B$, i.e.,

$$
L_{\mathcal{A}}=\left\{a \in \prod_{\wp \in \Pi(K)} L_{\wp} \mid a_{\wp} \in \mathcal{H}_{\wp} \text { for almost all } \wp \in U\right\} .
$$

As usual, this definition is independent of the choice of the order $\mathcal{H}$ on $L$, or the choice of the set $U$ of finite places. The rings $\Theta_{\mathcal{A}}$ and $\mathcal{A}=K_{\mathcal{A}}$ are defined analogously. We adopt the convention that $\mathcal{H}_{\wp}=L_{\wp}$ for $\wp \in S$, and define $\mathcal{H}_{\mathcal{A}}=\prod_{\wp \in \Pi(K)} \mathcal{H}_{\wp}$. The definition of $\mathcal{D}_{\mathcal{A}}$, for an order $\mathcal{D}$ on $\Theta$ is analogous. Let $J_{K}=\mathcal{A}^{*}$. Let $\phi: L \rightarrow \Theta$ be a representation of $L$ in a $K$-CSA $\Theta$. Then $\phi$ induces maps $L_{\wp} \rightarrow \Theta_{\wp}$ and $L_{\mathcal{A}} \rightarrow \Theta_{\mathcal{A}}$ which are also 
denoted $\phi$ by abuse of notation. Note that $\phi(\mathcal{H})$ is an order in $\Theta$, whence we can define the global spinor image, for any maximal $S$-order $\mathcal{D}$ on $\Theta$ containing $\phi(\mathcal{H})$, by either of the following equivalent formulas [3]:

$$
\begin{aligned}
H(\phi, \mathcal{D} \mid \mathcal{H}) & =\left\{N(a) \mid a \in \Theta_{\mathcal{A}}^{*}, a \phi\left(\mathcal{H}_{\mathcal{A}}\right) a^{-1} \subseteq \mathcal{D}_{\mathcal{A}}\right\} \\
H(\phi, \mathcal{D} \mid \mathcal{H}) & =J_{K} \cap \prod_{\wp \in \Pi(K)} H_{\wp}(\phi, \mathcal{D} \mid \mathcal{H})
\end{aligned}
$$

where $N$ is the reduced norm, and the local spinor image $H_{\wp}(\phi, \mathcal{D} \mid \mathcal{H})$ is defined by

$$
H_{\wp}(\phi, \mathcal{D} \mid \mathcal{H})=\left\{N(a) \mid a \in \Theta_{\wp}, a \phi\left(\mathcal{H}_{\wp}\right) a^{-1} \subseteq \mathcal{D}_{\wp}\right\} \subseteq K_{\wp}^{*} .
$$

In all that follows we assume that $\mathcal{D}$ is maximal. Note that, when $\phi(\mathcal{H})$ is contained in a second maximal order $\mathcal{D}^{\prime}=a \mathcal{D} a^{-1}$, then $H\left(\phi, \mathcal{D}^{\prime} \mid \mathcal{H}\right)=$ $a H(\phi, \mathcal{D} \mid \mathcal{H})$, and both sets contain the identity. In particular, both sets $H\left(\phi, \mathcal{D}^{\prime} \mid \mathcal{H}\right)$ and $H(\phi, \mathcal{D} \mid \mathcal{H})$ generate the same group $\Gamma(\phi, \mathcal{H})$. The class field $F=F_{-}(\phi, \mathcal{H})$ corresponding to $\Gamma(\phi, \mathcal{H}) K^{*}$ is called the lower representation field. This definition extends the one quoted in the introduction, since $F_{-}(\mathcal{H})=F_{-}(I d, \mathcal{H})$ when $I d: L \hookrightarrow \Theta$ is the identity. Let $\Sigma$ denote the spinor class field of maximal orders, and let $\rho: \Omega^{2} \rightarrow \operatorname{Gal}(\Sigma / K)$ be the distance function defined in $[3, \S 2]$, i.e., $\rho\left(\mathcal{D}, \mathcal{D}^{\prime}\right)=[N(a), \Sigma / K]$, where $\mathcal{D}^{\prime}=a \mathcal{D} a^{-1}$ and $t \mapsto[t, \Sigma / K]$ is the artin map on ideles. Then $F$ is the largest subfield satisfying

$$
\left.\phi(\mathcal{H}) \subseteq \mathcal{D}^{\prime} \Rightarrow \rho\left(\mathcal{D}, \mathcal{D}^{\prime}\right)\right|_{F}=i d
$$

There exists also an upper representation field $F^{\prime}=F^{-}(\phi, \mathcal{H})$ defined as the class field of

$$
\Delta(\phi, \mathcal{H})=\left\{a \in J_{K} \mid a H(\phi, \mathcal{D} \mid \mathcal{H}) K^{*}=H(\phi, \mathcal{D} \mid \mathcal{H}) K^{*}\right\}
$$

which is the smallest field satisfying

$$
\left.\rho\left(\mathcal{D}, \mathcal{D}^{\prime}\right)\right|_{F^{\prime}}=i d \Rightarrow \phi(\mathcal{H}) \subseteq a \mathcal{D}^{\prime} a^{-1} \text { for some } a \in \Theta
$$

These two fields coincide if and only if $H(\phi, \mathcal{D} \mid \mathcal{H}) K^{*}$ is a group, and in this case we call $F$ the representation field and say that the representation field is defined [1]. Next lemma gives an explicit description of $F_{-}(\phi, \mathcal{H})$ for any order $\mathcal{H}$ and any representation $\phi$. It follows by replacing $\mathcal{H}$ by $\phi(\mathcal{H})$ in $[4$, Lem.2.1]: 
Lemma 1 Let $\mathcal{H}$ be an order on a $K$-algebra $L$ and let $\phi: L \rightarrow$ $\Theta$ be a representation satisfying $\phi(\mathcal{H}) \subseteq \mathcal{D}$ for some maximal order $\mathcal{D}$ in the CSA $\Theta$. For every place $\wp \in U$, with maximal ideal $m_{\wp} \subseteq \mathcal{O}_{\wp}$ and residue field $\mathbf{K}_{\wp}$, we let $J_{\wp}$ be the only maximal two-sided ideal of $\mathcal{D}_{\wp}$ containing $m_{\wp} 1_{\mathcal{D}_{\wp}}$, and let $\mathbf{H}_{\wp}$ be the image of $\mathcal{H}_{\wp}$ in $\mathbf{D}_{\wp}=\mathcal{D}_{\wp} / J_{\wp}$. Let $\mathbf{E}_{\wp}$ be the center of the ring $\mathbf{D}_{\wp}$. Let $t_{\wp}$ be the greatest common divisor of the dimensions of the irreducible $\mathbf{E}_{\wp}$-representations of the $\mathbf{K}_{\wp}$-algebra $\mathbf{H}_{\wp}$. Then the lower representation field $F_{-}(\phi, \mathcal{H})$ is the maximal subfield $F$, of the spinor class field $\Sigma$, whose inertia degree $f_{\wp}(F / K)$ divides $t_{\wp}$ for every place $\wp$. Furthermore, if every irreducible $\mathbf{E}_{\wp}$-representation of $\mathbf{H}_{\wp}$ has dimension $t_{\wp}$, for every $\wp$, then the representation field is defined.

The residual algebras $\mathbf{H}_{\wp}$ in the previous lemma seem to depend heavily on the maximal order $\mathcal{D}$, but this is not the case.

Lemma 2 Let $\mathcal{H}$ be an order on a $K$-algebra $L$ and let $\phi: L \rightarrow \Theta$ be a faithful representation satisfying $\phi(\mathcal{H}) \subseteq \mathcal{D}$ for some maximal order $\mathcal{D}$ in the CSA $\Theta$. For every place $\wp \in U$, with maximal ideal $m_{\wp} \subseteq \mathcal{O}_{\wp}$ and residue field $\mathbf{K}_{\wp}$, the irreducible representations of the $\mathbf{K}_{\wp}$-algebra $\mathbf{H}_{\wp}$, defined as above, have exactly the same set of dimensions as the irreducible representations of the algebra $\widetilde{\mathbf{H}}_{\wp}=\mathcal{H}_{\wp} / m_{\wp} \mathcal{H}_{\wp}$.

Proof. It is immediate that $\mathbf{H}_{\wp}$ is a homomorphic image of $\widetilde{\mathbf{H}}_{\wp}$. It suffices therefore to note that any idempotent $T$ in $\widetilde{\mathbf{H}}_{\wp}$ can be lifted to an idempotent $t$ of $\mathcal{H}_{\wp}$ and $\pi_{\wp}^{-1} \phi(t)$ is not integral over $\mathcal{O}_{\wp}$, for any local uniformizing parameter $\pi_{\wp}$, whence the image of $T$ in $\mathbf{H}_{\wp}$ is never 0 .

The set of dimensions of the irreducible representation may change upon extending scalars to $\mathbf{E}_{\wp}$ as in Lemma 1 but, as follows from [4, Lemma 3.1], the last condition in Lemma 1 is preserved by extension of scalars, and it holds therefore for every representation of the algebra $L=K \mathcal{H}$, as long as every irreducible representation of $\widetilde{\mathbf{H}}_{\wp}$ has the same dimension.

Example When $\Theta$ is a quaternion algebra, The residual algebra $\mathbf{D}_{\wp}$ is either a quadratic extension $\mathbf{L}_{\wp}$, of the residue field $\mathbf{K}_{\wp}$, or a matrix algebra $\operatorname{Mat}_{2}\left(\mathbf{K}_{\wp}\right)$. Then, either $\mathbf{H}_{\wp} \subseteq \mathbf{D}_{\wp}$ has a unique two-dimensional irreducible representation or just one-dimensional representations. In either case the last condition in Lemma 1 is satisfied, so the representation 
field exists for all representations in quaternion algebras. By Lemma 2, the same holds for an arbitrary representation of an order contained in a quaternion algebra.

Example Let $Q$ be a quaternion division algebra ramifying at a place $\wp$, and let $L$ be a maximal subfield that is inert (unramified) at $\wp$. Let $\Theta=$ $\operatorname{Mat}_{2}(Q)$. Let $\omega$ be an integral generator for $L / K$ satisfying $\mathcal{O}_{L_{\wp}}=\mathcal{O}_{K_{\wp}}[\omega]$ and consider the embeddings $\phi_{1}, \phi_{2}: \mathcal{H} \subseteq L \rightarrow \Theta$ defined by

$$
\phi_{1}(\omega)=\left(\begin{array}{cc}
\omega & 0 \\
0 & \omega
\end{array}\right), \quad \phi_{2}(\omega)=\left(\begin{array}{cc}
\bar{\omega} & 0 \\
0 & \omega
\end{array}\right),
$$

where the bar denotes the usual involution. Then, if $\mathcal{H}=\mathcal{O}_{L}$, the residual algebra $\widetilde{\mathbf{H}}_{\wp}$ is the unique quadratic extension $\mathbf{L}_{\wp}$ of the residue field $\mathbf{K}_{\wp}$. It follows that $\mathbf{H}_{\wp} \cong \mathbf{L}_{\wp}$ in each case, but $\mathbf{E}_{\wp} \mathbf{H}_{\wp} \cong \mathbf{L}_{\wp}$ in the first case, while $\mathbf{E}_{\wp} \mathbf{H}_{\wp} \cong \mathbf{L}_{\wp} \times \mathbf{L}_{\wp}$ in the second.

Remark This section can be extended, word-by-word, to orders over an $A$-curve $X$, as defined in [3], with structure sheaf $\mathcal{O}=\mathcal{O}_{X}$ and field of rational functions $K=K(X)$. In other words, all results here apply to $X$-orders for a projective curve $X$ over a finite field, as defined in [8]. This latter setting is called the projective case in all that follows. Let $|X|$ be the set of closed points of $X$. Note that the set $|X|$ of closed points can be identified with the set $\Pi(K)$ of places of $K$ defined above. We set $U=|X|$ and $S=\emptyset$ in the projective case, i.e.,there are no infinite places. In this case we define the maximal ideal $I^{\wp}$ corresponding to a place $\wp$ as the one-dimensional lattice (sheaf) on $K$ satisfying

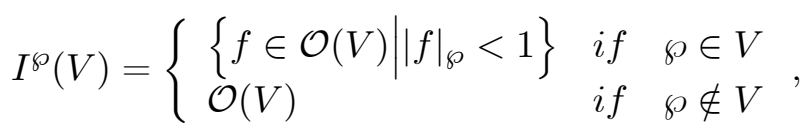

for any open set $V \subseteq U$. An $S$-order $\mathcal{H}$ on a finite dimensional $K$-algebra $L$ is an $X$-order, i.e., a locally free sheaf of $\mathcal{O}$-algebras, whose generic fiber is $L$, i.e., $L=K \otimes_{\mathcal{O}} \mathcal{H}[8]$. Lattices and ideals must be interpreted similarly in this case. Certainly, strong approximation with respect to $S=\emptyset$ never holds, so spinor genera do not coincide with conjugacy classes in this context, and in fact the number of conjugacy classes of maximal orders can 
be infinite (see the example at the end of $\S 5$ ), but two orders $\mathcal{D}$ and $\mathcal{D}^{\prime}$ in the same spinor genus have conjugate rings of sections $\mathcal{D}(V)$ and $\mathcal{D}^{\prime}(V)$ for every open set $V$ outside a finite set. The image of an order $\mathcal{H}$ on $L$ under a representation $\phi: L \rightarrow \Theta$ is the sheaf defined by $\phi(\mathcal{H})(V)=\phi(\mathcal{H}(V))$.

\section{Proof of Theorem 1 and Theorem 2.}

In all of this section we let $E$ be a finite dimensional algebra over a local field $k$. We let $C$ be a $k$-CSA, and we fix a representation $\phi: E \rightarrow C$. Let $\mathcal{E}$ be a (local) order in $E$. Recall that $C$ is isomorphic to a matrix algebra $\operatorname{Mat}_{f}(B)$ over a division algebra $B$, so we can assume $C=\operatorname{Mat}_{f}(B)$. In particular, the space of column vectors $B^{f}$ is a $(E, B)$-bimodule $(\S 2)$.

In all that follows, we use the definition

$$
H_{k}(\phi, \mathcal{D} / \mathcal{H})=\left\{N(a) \mid a \in \operatorname{Mat}_{f}(B), a \phi(\mathcal{H}) a^{-1} \subseteq \mathcal{D}\right\}
$$

for any maximal order $\mathcal{D} \subseteq \operatorname{Mat}_{f}(B)$ and any order $\mathcal{H} \subseteq E$. This is consistent with our previous notation in the sense that $H_{\wp}(\phi, \widetilde{\mathcal{D}} / \widetilde{\mathcal{H}})=$ $H_{K_{\wp}}\left(\phi, \widetilde{\mathcal{D}}_{\wp} / \widetilde{\mathcal{H}}_{\wp}\right)$, when $\widetilde{\mathcal{D}}$ and $\widetilde{\mathcal{H}}$ are global orders.

Lemma 3 Let $\mathcal{E}$ be an order in the $k$-algebra $E$, where $k$ is a local field. Let $\phi: E \rightarrow M_{f}(B)$ be a representation, and let $\{0\}=M_{0} \subseteq$ $\ldots \ldots \subseteq M_{r}=B^{f}$ be a composition series of the corresponding $(E, B)$ bimodule. Let $C_{i}$ be the algebra of right-B-linear maps in $M_{i} / M_{i-1}$, and let $\phi_{i}: L \rightarrow C_{i}$ denote the corresponding subrepresentation. Then there exist a family of maximal orders $\left\{\mathcal{D}_{i}\right\}$, with $\phi_{i}(\mathcal{E}) \subseteq \mathcal{D}_{i} \subseteq C_{i}$, and a maximal order $\mathcal{D} \subseteq C$, containing $\phi(\mathcal{E})$, such that

$$
H(\phi, \mathcal{D} \mid \mathcal{E}) \supseteq \prod_{i=1}^{r} H\left(\phi_{i}, \mathcal{D}_{i} \mid \mathcal{E}\right) .
$$

Proof. By the remarks at the begining of $\S 2$, there exists a basis of $B^{f}$ for which the algebra $L=\phi(E)$ is contained in the ring of matrices with a block decomposition of the form (2.1). Choose $\mathcal{D}_{i}$, for $i=1, \ldots, r$ (resp. $\mathcal{D})$, as the rings of integral matrices in the corresponding basis of $M_{i} / M_{i-1}$ (resp. $\left.B^{f}\right)$. Let $\pi=\pi_{B}$ be a uniformizing parameter. Note that, whenever 
$u_{i}$ is a generator for $\mathcal{D}_{i} \mid \phi_{i}(\mathcal{E})$, as defined ${ }^{1}$ in $[3, \S 2]$, then

$$
u=\left(\begin{array}{cccc}
\pi^{f N} u_{1} & 0 & \cdots & 0 \\
0 & \pi^{2 f N} u_{2} & \cdots & 0 \\
\vdots & \vdots & \ddots & \vdots \\
0 & 0 & \vdots & \pi^{t f N} u_{r}
\end{array}\right)
$$

is a generator for $\mathcal{D} \mid \phi(\mathcal{E})$ when $N$ is large enough, so that the block $\pi^{f N} a_{i j}(h)$ is integral, for every $i<j$ and every $h$ in the compact set $\mathcal{E}$. Note also that the reduced norm of $u$ satisfies

$$
N(u) \mathcal{O}_{k}^{*} k^{* f}=\left(\prod_{i=1}^{r} N\left(u_{i}\right)\right) \mathcal{O}_{k}^{*} k^{* f},
$$

whence the result follows.

Proposition 1 Let $K$ be a global field, $L$ a $K$-algebra, $\Theta$ a K-CSA, and $\phi: L \rightarrow \Theta$ a representation. Let $S$ be a non-empty finite set of places in $K$ containing the archimedean places if any, and let $\mathcal{H} \subseteq L$ be an $S$-order. Assume that, for each irreducible component $\phi_{i}, i=1, \ldots, r$, of the representation $\phi: L \rightarrow \Theta$, the representation field $F\left(\phi_{i}, \mathcal{H}\right)$ is defined. Then the representation field $F(\phi, \mathcal{H})$ is defined, and in fact $F(\phi, \mathcal{H})=\bigcap_{i} F\left(\phi_{i}, \mathcal{H}\right)$.

Proof. Choose the orders $\mathcal{D}$ and $\mathcal{D}_{i}$, for $i=1, \ldots, r$, as in the preceding lemma. It suffices to prove that

$$
H_{\wp}(\phi, \mathcal{D} \mid \mathcal{H})=\prod_{i=1}^{r} H_{\wp}\left(\phi_{i}, \mathcal{D}_{i} \mid \mathcal{H}\right)
$$

locally at every place $\wp \notin S$. One contention follows from Lemma 3 . The other contention follows from Lemma 1 if we note that the irreducible representations of the residual algebra $\widehat{\mathbf{H}}_{\wp}=\phi\left(\mathcal{H}_{\wp}\right) / \pi \phi\left(\mathcal{H}_{\wp}\right)$ correspond to the irreducible representations of the residual algebras $\widehat{\mathbf{H}}_{i, \wp}=\phi_{i}\left(\mathcal{H}_{\wp}\right) / \pi \phi_{i}\left(\mathcal{H}_{\wp}\right)$ for $i=1, \ldots, r$, by the proof of Lemma 2. The result follows.

Theorem 1 and Theorem 2 follow from next corollary:

\footnotetext{
${ }^{1}$ This use of the word generator is taken from the theory of representations of integral quadratic forms, see for example $[11, \S 2]$.
} 
Corollary 1 Let $K, L, \Theta, \phi, S$, and $\mathcal{H}$ be as in the preceding proposition. If every irreducible component $\psi: L \rightarrow \Theta^{\prime}$ of the representation $\phi: L \rightarrow \Theta$ satisfies any of the following conditions:

1. $\psi(L)$ is a quaternion algebra, or

2. $\psi(L)$ is commutative,

then the spinor class field is defined.

Proof. It suffices to prove that either hypotheses implies the last condition in Lemma 1. This follows as in the proof of [3, Proposition 4.3] when $\psi(L)$ is commutative. When $\psi(L)$ is contained in a quaternion algebra, this follows from Lemma 2, an the example following it.

\section{Proof of Theorem 3.}

Let $k=K_{\wp}$ be a local field, let $E / k$ be the unique unramified quadratic extension, and let $L=\operatorname{Mat}_{n}(E)$. Note that there exists, up to change of basis, a unique faithful representation $\phi: L \rightarrow M_{2 n}(k)$ and it can be realized by identifying $E^{n}$ with $k^{2 n}$. Moreover, the basis can be chosen in a way that $\mathcal{O}_{E}^{n}$ is identified with $\mathcal{O}_{k}^{2 n}$. In this case we say that the representation is integral. Next result is now immediate:

Lemma 4 Let $E / k$ be an unramified quadratic extension of local fields. If $\phi: \operatorname{Mat}_{n}(E) \rightarrow M_{2 n}(k)$ is a faithful integral representation, for any vector $v$ in $\mathcal{O}_{k}^{2 n} \backslash \pi \mathcal{O}_{k}^{2 n}$ we have $\phi\left(\operatorname{Mat}_{n}\left(\mathcal{O}_{E}\right)\right) v=\mathcal{O}_{k}^{2 n}$.

Until the end of this section, we consider the order

$$
\mathcal{H}=\left(\begin{array}{cc}
\mathcal{O}_{k} 1_{E} & \mathcal{O}_{E} \\
0 & \mathcal{O}_{E}
\end{array}\right)+\pi\left(\begin{array}{cc}
\mathcal{O}_{E} & \mathcal{O}_{E} \\
\mathcal{O}_{E} & \mathcal{O}_{E}
\end{array}\right) \subseteq \operatorname{Mat}_{2}(E)
$$

where $\pi$ is a uniformizing parameter of $k$, or $E$. 
Lemma 5 Let $L=M_{2}(E)$ for an unramified quadratic extension $E / k$, let $\mathcal{H}$ be as in (4.1), and let $\phi: L \rightarrow$ Mat $_{4}(k)$ be a faithful integral representation. Let $\mathcal{D}=\operatorname{Mat}_{4}\left(\mathcal{O}_{k}\right)$. Then the relative spinor image is

$$
H_{k}(\phi, \mathcal{D} \mid \mathcal{H})=k^{* 4} \mathcal{O}_{k}^{*} \cup\left(\pi^{2} k^{* 4} \mathcal{O}_{k}^{*}\right) \cup\left(\pi^{3} k^{* 4} \mathcal{O}_{k}^{*}\right)
$$

Proof. To simplify notations, we identify $\mathcal{H}$ with $\phi(\mathcal{H})$, i.e., we assume $\phi$ is the identity. Note that $a \mathcal{H} a^{-1} \subseteq \mathcal{D}$ if and only if $\mathcal{H} \subseteq a^{-1} \mathcal{D} a$, and the statements $d \in \mathcal{D}$ and $d^{\prime} \in \mathcal{D}^{\prime}=a^{-1} \mathcal{D} a$ are equivalent to $d \mathcal{O}_{k}^{4}=\mathcal{O}_{k}^{4}$ and $d^{\prime} a^{-1} \mathcal{O}_{k}^{4}=a^{-1} \mathcal{O}_{k}^{4}$, respectively. It follows that $H_{k}(\phi, \mathcal{D} \mid \mathcal{H})$ is the set of norms of elements $a$ for which $a^{-1} \mathcal{O}_{k}^{4}$ is invariant under $\mathcal{H}$. Let $M$ be a lattice that is invariant under $\mathcal{H}$. Multiplying by a power of $\pi$ if needed, we can assume that $M$ is contained in $\mathcal{O}_{k}^{4}$, but not $\pi \mathcal{O}_{k}^{4}$. Note that $M$ is also invariant under every sub-order or ideal in $\mathcal{H}$ :

1. Since $M$ is invariant under $\pi M a t_{2}\left(\mathcal{O}_{E}\right)$, a direct application of Lemma 4 shows that $M$ contains $\pi \mathcal{O}_{k}^{4}$.

2. The order $\mathcal{H}_{1}=\left(\begin{array}{cc}\mathcal{O}_{k} 1_{E} & 0 \\ 0 & \mathcal{O}_{E}\end{array}\right)$ can only stabilize lattices of the form $\Lambda_{1} \times \Lambda_{2}$ with $\Lambda_{2}=\pi^{r} \mathcal{O}_{k}^{2}$ (set $n=1$ in Lemma 4$)$.

3. The order $\mathcal{H}_{2}=\left(\begin{array}{cc}\mathcal{O}_{k} 1_{E} & \mathcal{O}_{E} \\ 0 & \mathcal{O}_{k} 1_{E}\end{array}\right)$ can only stabilize lattices of the form $\Lambda_{1} \times \Lambda_{2}$ with $\mathcal{O}_{E} \Lambda_{2} \subseteq \Lambda_{1}$.

Let $\mathbf{Z}$ denote the ring of rational integers. We define the local distance between $\mathcal{D}_{1}$ and $\mathcal{D}_{2}=b \mathcal{D}_{1} b^{-1}$ by

$$
\rho\left(\mathcal{D}_{1}, \mathcal{D}_{2}\right)=v(N(b))+4 \mathbf{Z} \in \mathbf{Z} / 4 \mathbf{Z}
$$

where $N: \operatorname{Mat}_{4}(k)^{*} \rightarrow k^{*}$ is the determinant, and $v$ denotes the valuation on $k$, normalized in a way that $v(\pi)=1$. Note that $\rho$ is well defined, since the normalizer ${ }^{2}$ of $\mathcal{D}_{1}$ is $k^{*} \mathcal{D}_{1}^{*}$, and its set of norms is $k^{* 4} \mathcal{O}_{k}^{*}$. From what precedes, any $\mathcal{H}$-invariant lattice as above has the form $M=\Lambda_{1} \times \Lambda_{2}$, with $\Lambda_{1}$ and $\Lambda_{2}$ of rank $2, \pi \mathcal{O}_{k}^{4} \subset M \subseteq \mathcal{O}_{k}^{4}$, and falls into one of the following cases:

\footnotetext{
${ }^{2}$ Since $\mathcal{D}_{1}$ is not a subgroup, some authors prefer the term conjugation-stabilizer.
} 
1. If $\Lambda_{2} \neq \pi \mathcal{O}_{k}^{2}$, then necessarily $\Lambda_{2}=\mathcal{O}_{k}^{2}$, and in that case $\Lambda_{1}=\mathcal{O}_{k}^{2}$, so that $M=\mathcal{O}_{k}^{4}$, and $\rho\left(\mathcal{D}, \mathcal{D}^{\prime}\right)=\overline{0} \in \mathbf{Z} / 4 \mathbf{Z}$.

2. If $\Lambda_{2}=\pi \mathcal{O}_{k}^{2}$, then $\Lambda_{1}$ is an arbitrary lattice satisfying $\pi \mathcal{O}_{k}^{2} \subseteq \Lambda_{1} \subseteq$ $\mathcal{O}_{k}^{2}$. There are three subcases:

1. If $\Lambda_{1}=\pi \mathcal{O}_{k}^{2}$ we have $\rho_{k}\left(\mathcal{D}, \mathcal{D}^{\prime}\right)=\overline{0}$.

2. If $\Lambda_{1}=\mathcal{O}_{k}^{2}$ we have $\rho_{k}\left(\mathcal{D}, \mathcal{D}^{\prime}\right)=\overline{2}$.

3. If $\Lambda_{1}=\mathcal{O}_{k} v+\pi \mathcal{O}_{k}^{2}$, for some $v \in \mathcal{O}_{k}^{2} \backslash \pi \mathcal{O}_{k}^{2}$, we have $\rho_{k}\left(\mathcal{D}, \mathcal{D}^{\prime}\right)=\overline{3}$.

The result follows.

Proof of Theorem 3. Let $H$ be the Hilbert class field of $K$. Let $\Sigma$ be the spinor class field of maximal orders in $\operatorname{Mat}_{4}(K)$. Then $\Sigma$ is the maximal sub-extension of $H$ of exponent 4 , and in particular, the Galois group $\operatorname{Gal}(\Sigma / K)$ has an element $\sigma$ of order 4 . Let $\wp$ be a place satisfying $\left|\left[I_{\wp}, \Sigma / K\right]\right|=\sigma$, where $I_{\wp}$ is the maximal ideal corresponding to $\wp$, and $I \mapsto|[I, \Sigma / K]|$ denotes the artin map on ideals. Let $F^{\prime}$ be a degree-4 unramified cyclic extension of $K$ such that $f_{\wp}\left(F^{\prime} / K\right)=4$, and let $F / K$ be the unique quadratic sub-extension. We let $\mathcal{H}_{\wp}$ be defined as in equation (4.1) with $E=F \otimes_{K} K_{\wp}$, while we let $\mathcal{H}$ be maximal in $\operatorname{Mat}_{2}(F)$ at all other places. It is immediate from Lemma 4 that any maximal order of $\operatorname{Mat}_{2}(F)$ is contained in a unique maximal order of $\operatorname{Mat}_{4}(K)$ at inert places $\wp^{\prime} \neq \wp$ for $F / K$, whence

$$
H_{\wp^{\prime}}(\phi, \mathcal{D} \mid \mathcal{H})=K_{\wp^{\prime}}^{* 4} \mathcal{O}_{\wp^{\prime}}^{*},
$$

at those places. On the other hand, at places $\wp^{\prime \prime} \neq \wp$ splitting $F / K$, every invariant lattice has the form $\pi_{\wp^{\prime \prime}}^{t} \mathcal{O}_{\wp^{\prime \prime}}^{2} \times \pi_{\wp^{\prime \prime}}^{s} \mathcal{O}_{\wp^{\prime \prime}}^{2}$, for a suitable uniformizing parameter $\pi_{\wp^{\prime \prime}}$ of $K_{\wp^{\prime \prime}}$, whence

$$
H_{\wp^{\prime \prime}}(\phi, \mathcal{D} \mid \mathcal{H})=K_{\wp^{\prime \prime}}^{* 2} \mathcal{O}_{\wp^{\prime \prime}}^{*}
$$

As $f_{\wp^{\prime \prime}}\left(F^{\prime} / K\right) \leq 2$ at the latter places, the Artin symbol of every element in $H_{\tilde{\wp}}(\phi, \mathcal{D} \mid \mathcal{H})$ is trivial on $F^{\prime}$ for every place $\tilde{\wp} \neq \wp$. It follows that the image in $\operatorname{Gal}\left(F^{\prime} / K\right)$ of $H(\phi, \mathcal{D} \mid \mathcal{H})$ is the set

$$
\left\{i d,\left[\wp, F^{\prime} / K\right]^{2},\left[\wp, F^{\prime} / K\right]^{3}\right\} .
$$

We conclude that the upper representation field $F^{-}(\phi, \mathcal{H})$ contains $F^{\prime}$, while the lower representation field $F_{-}(\phi, \mathcal{H})$ intersects $F^{\prime}$ trivially. The result follows. 
Remark Assume we have a rank- 8 order $\mathcal{H}$, and a representation $\phi$, for which the representation field is not defined. By Proposition 1, we can assume that the representation $\phi$ is irreducible (and faithfull). Furthermore, $\mathcal{H}$ cannot be commutative. We conclude that $L=K \mathcal{H}$ is a quaternion algebra over a quadratic extension $E / K$. In particular, we conclude that the representation field is defined for all orders of non-maximal rank in a 9-dimensional CSA.

\section{Applications and examples}

For every integer $n \geq 3$, we constructed in [2] an order in $\operatorname{Mat}_{n}(K)$, for a suitable number field $K$, whose representation field is not defined ${ }^{3}$ (see also [3, Ex.3.6]). We choose $\mathcal{H}_{\wp}$, locally at some place $\wp$, as the pre-image in $\operatorname{Mat}_{n}\left(\mathcal{O}_{\wp}\right)$ of the residual algebra

$$
\mathbf{H}_{\wp}=\left(\begin{array}{cc}
\mathbf{K} & \mathbf{K} \\
0 & \operatorname{Mat}_{n-1}(\mathbf{K})
\end{array}\right), \quad \mathbf{K}=\mathcal{O}_{K} / I_{\wp},
$$

where $I_{\wp}$ is the global prime ideal corresponding to $\wp$, and we showed that the residual algebra $\mathbf{H}_{\wp}$ alone is not sufficient to determine wether the spinor image $H_{\wp}(\phi, \mathcal{D} \mid \mathcal{H})$ is a group. In fact, here we can prove a stronger statement:

Proposition 2 Let $k$ be a local field with ring of integers $\mathcal{O}_{k}$ and maximal ideal $m_{k}$. Let $E$ be a division $k-C S A$, and let $\mathcal{D}=\operatorname{Mat}_{f}\left(\mathcal{O}_{E}\right) \subseteq$ $\operatorname{Mat}_{f}(E)$. Let $\mathbf{E}=\mathcal{O}_{E} / m_{E}$, where $m_{E}$ is the unique maximal bilateral ideal of $\mathcal{O}_{E}$. For every residual algebra $\mathbf{H} \subseteq \operatorname{Mat}_{f}(\mathbf{E})$, there exists a local order $\mathcal{H} \subseteq \mathcal{D}$ of maximal rank whose image in $M_{a}(\mathbf{E})$ is $\mathbf{H}$ and whose relative local spinor image $H_{k}(i d, \mathcal{D} \mid \mathcal{H})$ is a group.

Proof. Let $M_{1} \subseteq M_{2} \subseteq \cdots \subseteq M_{r}$ be a maximal flag of $(\mathbf{H}, \mathbf{E})$-bimodules in $\mathbf{E}^{f}$, and choose a basis such that $\mathbf{H}$ is contained in the ring of matrices of $\operatorname{Mat}_{f}(\mathbf{E})$ with a block decomposition of the form (2.1). Lift $\mathbf{H}$ to a local order $\mathcal{H}^{\prime}$ which also consists only on matrices with a block decomposition

\footnotetext{
${ }^{3}$ The fact that the algebra is globally split is not essential here. We have only assumed it for simplicity.
} 
of the form (2.1) for a suitable basis, so in particular, whenever $u_{i}$ is a generator for $\mathcal{D}_{i} \mid \mathcal{H}_{i}$, then the matrix $u$ as in (3.1) is a generator for $\mathcal{D} \mid \mathcal{H}^{\prime}$ as soon as $N$ is big enough. Note also that, for every irreducible representation $\phi_{i}$ corresponding to this flag, the residual algebra $\phi_{i}(\mathcal{H}) / m_{k} \phi_{i}(\mathcal{H})$ has a unique irreducible representation, hence the representation field $F\left(\mathcal{H}^{\prime}\right)$ is defined. Now we choose a finite number of generators for $\mathcal{D} \mid \mathcal{H}^{\prime}$ whose reduced norms form a set of representatives for $H_{k}\left(i d, \mathcal{D} \mid \mathcal{H}^{\prime}\right) / k^{* 2}$, and note that they are also generators for $\mathcal{D} \mid \mathcal{H}$, where $\mathcal{H}=\mathcal{H}^{\prime}+\pi^{M} \mathcal{D}$, for a uniformizing parameter $\pi$ of $k$, if $M$ is chosen big enough. It follows that, if $\Gamma_{k}(i d, \mathcal{H})$ is the group generated by $H_{k}(i d, \mathcal{D} \mid \mathcal{H})$, and $\Gamma_{k}\left(i d, \mathcal{H}^{\prime}\right)$ is defined analogously, then

$$
\Gamma_{k}(i d, \mathcal{H})=\Gamma_{k}\left(i d, \mathcal{H}^{\prime}\right)=H_{k}\left(i d, \mathcal{D} \mid \mathcal{H}^{\prime}\right) \subseteq H_{k}(i d, \mathcal{D} \mid \mathcal{H}) \subseteq \Gamma_{k}(i d, \mathcal{H})
$$

where the first identity holds since $\Gamma_{k}(\phi, \mathcal{H})$, when $\phi$ is faithfull, depends only on the residual algebra $\mathbf{H}$ (and the field $\mathbf{E}$ ) by Lemma 2. The result follows.

Next result is a straightforward consequence of Proposition 1:

Proposition 3 Let $K$ be a global field, $L$ a K-algebra, $\Theta$ a K-CSA, and $\phi: L \rightarrow \Theta$ a representation. Let $\phi^{\prime}: L \rightarrow \operatorname{Mat}_{n}(\Theta)$ be the composition of $\phi$ with the cannonical embedding of $\Theta$ into $\operatorname{Mat}_{n}(\Theta)$, and let $\mathcal{D} \subseteq \Theta$ and $\mathcal{D}^{\prime} \subseteq \operatorname{Mat}_{n}(\Theta)$ be maximal orders containing the respective images of $\mathcal{H}$. Then the representation field $F\left(\phi^{\prime}, \mathcal{D}^{\prime} \mid \mathcal{H}\right)$ is defined whenever $F(\phi, \mathcal{D} \mid \mathcal{H})$ is defined, an in this case they are equal.

In fact, for commutative algebras ${ }^{4}$, we can prove the following partial generalization:

Proposition 4 Let $K$ be a global field, $L$ a commutative $K$-algebra, let $\Theta$ and $\Theta^{\prime}$ be two K-CSAs of relatively prime order, and let $\phi: L \rightarrow \Theta$ a representation. Let $\phi^{\prime}=\phi \otimes i d: L \cong L \otimes_{K} K \rightarrow \Theta \otimes_{K} \Theta^{\prime}$, and let $\mathcal{D} \subseteq \Theta$ and $\mathcal{D}^{\prime} \subseteq \Theta \otimes_{K} \Theta^{\prime}$ be maximal orders containing the respective images of $\mathcal{H}$. Assume that $\operatorname{dim}_{K}\left(\Theta^{\prime}\right)$ is odd. Then $F\left(\phi^{\prime}, \mathcal{D}^{\prime} \mid \mathcal{H}\right)=F(\phi, \mathcal{D} \mid \mathcal{H})$.

Proof. It suffices to prove that $H_{\wp}(\phi, \mathcal{D} \mid \mathcal{H})=H_{\wp}\left(\phi^{\prime}, \mathcal{D}^{\prime} \mid \mathcal{H}\right)$ at all places. At complex infinite places there is nothing to prove. At real infinite places

\footnotetext{
${ }^{4}$ Actually, any condition that guarantees the existence of the representation field can replace commutativity here.
} 
$\wp$, the odd-dimensional R-CSA $\Theta_{\wp}^{\prime}$ is a matrix algebra, so the result is immediate. Assume now that $\wp$ is a finite place. By the proof of Proposition 1 we can assume $\Theta_{\wp}^{\prime}=B_{\wp}^{\prime}$ is a division algebra with residue field $\mathbf{B}_{\wp}^{\prime}$, and $\phi$ is irreducible, so $\phi\left(L_{\wp}\right)$ is a field. We can assume $L_{\wp}=\phi\left(L_{\wp}\right)$. The semisimple part of the residual algebra $\mathbf{H}_{\wp}$ in Lemma 1 is the residue field $\mathbf{L}_{\wp}$ of $L_{\wp}$. In particular, if $\Theta_{\wp}=\operatorname{Mat}_{f}\left(E_{\wp}\right)$, where $E_{\wp}$ is a division algebra, every irreducible $\mathbf{E}_{\wp}$-representation of $\mathbf{H}_{\wp} \mathbf{E}_{\wp}$, where $\mathbf{E}_{\wp}$ is the residue field of $E_{\wp}$, has dimension $\left[\mathbf{E}_{\wp} \mathbf{L}_{\wp}: \mathbf{E}_{\wp}\right]$. Note that $\mathbf{B}_{\wp}^{\prime}$ is an extension of the residue field $\mathbf{K}_{\wp}$ of $K_{\wp}$ whose degree is relatively prime to $\operatorname{dim}_{K}(\Theta)$. Furthermore, $\Theta_{\wp} \otimes_{K_{\wp}} \Theta_{\wp}^{\prime} \cong \operatorname{Mat}_{f}\left(E_{\wp} \otimes_{K_{\wp}} B_{\wp}^{\prime}\right)$, and $E_{\wp} \otimes_{K_{\wp}} B_{\wp}^{\prime}$ is a division algebra with residue field $\mathbf{E}_{\wp}^{\prime}=\mathbf{E}_{\wp} \otimes_{\mathbf{K}_{\wp}} \mathbf{B}_{\wp}^{\prime}$. Replacing $\mathbf{E}_{\wp}$ by $\mathbf{E}_{\wp}^{\prime}$ in Lemma 1 has the effect of replacing $\left[\mathbf{E}_{\wp} \mathbf{L}_{\wp}: \mathbf{E}_{\wp}\right]$. by $\left[\mathbf{E}_{\wp}^{\prime} \mathbf{L}_{\wp}: \mathbf{E}_{\wp}^{\prime}\right]$, but these degrees are equal by the hipotheses on the dimension of the algebras.

Example In [10], examples of commutative orders that are selective on indefinite quaternion division algebras, were explicitly computed. If we follow [12], and call an order selective, when it is contained in some but not all the maximal orders in a given central simple algebra, the existence of selective orders on division algebras of dimension $n^{2}$ whenever $n$ is twice an odd number is a consequence of Proposition 4. In [6] we proved that, in fact, all selectivity in division algebras arises in this way from quaternion subalgebras.

Example The condition on the dimensions of the algebras in Proposition 4 is necessary. In fact, If $L / K$ is a quadratic extension and $\mathcal{H}=\mathcal{O}_{L}$ is a selective suborder in a quaternion algebra $\Theta$, the same proof as above shows that $\mathcal{H}$ is no longer selective as a suborder of $\Theta \otimes_{K} \Theta^{\prime}$ for any quaternion algebra $\Theta^{\prime}$ that ramifies at a place that is inert for $L$.

Example Let $\mathcal{H}$ and $\mathcal{H}^{\prime}$ be global orders in $\operatorname{Mat}_{n}(K)$ of the form

$$
\mathcal{H}=\left(\begin{array}{cccc}
\mathcal{H}_{1} & M_{12} & \cdots & M_{1 r} \\
0 & \mathcal{H}_{2} & \cdots & M_{2 r} \\
\vdots & \vdots & \ddots & \vdots \\
0 & 0 & \cdots & \mathcal{H}_{r}
\end{array}\right), \quad \mathcal{H}^{\prime}=\left(\begin{array}{cccc}
\mathcal{H}_{1} & 0 & \cdots & 0 \\
0 & \mathcal{H}_{2} & \cdots & 0 \\
\vdots & \vdots & \ddots & \vdots \\
0 & 0 & \cdots & \mathcal{H}_{r}
\end{array}\right)
$$

The results in this work show that whenever $\mathcal{H}^{\prime}$ embeds into every maximal order (and $S \neq \emptyset$ ), so does $\mathcal{H}$. In fact, using strong approximation, it 
is easy to construct a sequence of global conjugates of $\mathcal{H}$ whose adelization converges in the Hausdorff topology to $\mathcal{H}^{\prime}$, whence a similar result holds for any genus of orders of maximal rank. This fails to hold in the projective case (see the remark at the end of $\S 2$ and the following example). However, in this case we still have an analogous result in terms of spinor genera.

Example Let $\mathcal{H}$ be the order

$$
\mathcal{H}=\left(\begin{array}{cc}
\mathcal{O} & \mathcal{I} \\
0 & \mathcal{O}
\end{array}\right),
$$

for an arbitrary ideal $\mathcal{I}$. Then $\mathcal{H}$ is contained in an order of every spinor genus of maximal orders in $\operatorname{Mat}_{2}(K)$, as in the preceding example. On the other hand, in the projective case (see the las remark in $\S 2$ ), there exists conjugacy classes of maximal $X$-orders that fail to contain a copy of $\mathcal{H}$. For example, if for a fixed divisor $D$ on $X$, we denote by $\mathcal{I}=\mathcal{L}_{D}$ the sheaf defined on open subsets $V \subseteq X$ by

$$
\mathcal{L}_{D}(V)=\left\{f \in K|\operatorname{div}(f)|_{V} \geq-\left.D\right|_{V}\right\}
$$

then the ring of global sections $\mathcal{H}(X)$ is the set of all matrices of the form $\left(\begin{array}{ll}a & f \\ 0 & b\end{array}\right)$ where $a$ and $b$ are constants and $f \in \mathcal{L}_{D}(X)$. The dimension of $\mathcal{L}_{D}(X)$ grows with the degree of $D$ acording to Riemann-Roch's Theorem, whence by chosing a divisor $D$ of sufficiently large degree, we can assume that $\mathcal{H}$ cannot be embedded in any order of an arbitrary prescribed finite family.

\section{References}

[1] L. Arenas-Carmona, Applications of spinor class fields: embeddings of orders and quaternionic lattices, Ann. Inst. Fourier (Grenoble) 53, pp. 2021-2038, (2003).

[2] L. Arenas-Carmona. Relative spinor class fields: A counterexample, Archiv. Math. 91, pp. 486-491, (2008). 
[3] L. Arenas-Carmona, Spinor class fields for commutative orders, Ann. Inst. Fourier (Grenoble) 62, pp. 807-819, (2012).

[4] L. Arenas-Carmona, Spinor class fields for cyclic orders, Acta Arith. 156, pp. 143-156, (2012).

[5] L. Arenas-Carmona, Computing quaternion quotient graphs via representations of orders, J. Algebra. 402, pp. 258-279, (2014).

[6] L. Arenas-Carmona, Selectivity in division algebras, Archiv Math. 103, pp. 139-146, (2014).

[7] L. Arenas-Carmona, Roots of unity in definite quaternion orders, Acta Arith. 170, pp. 381-393, (2015).

[8] J. Brzezinski, Riemann-Roch Theorem for locally principal orders, Math. Ann., 276, pp. 529-536, (1987).

[9] C. Chevalley, L'arithmétique sur les algèbres de matrices, Herman: Paris (1936).

[10] T. Chinburg and E. Friedman, An embedding theorem for quaternion algebras, J. London Math. Soc. 60.2 (1999), 33-44.

[11] J.S. Hsia, Y. Y. Shao, and F. Xu, Representations of indefinite quadratic forms, J. Reine Angew. Math. 494, pp. 129-140, (1998).

[12] B. Linowitz and T. Shemanske, Embedding orders in central simple algebras, J. Théor. Nombres Bordeaux 24, pp. 405-424, (2012).

[13] M. PAPIKIAN, Local Diophantine properties of modular curves of Delliptic sheaves, J. Reine Angew. Math. 664, pp. 115-140, (2012).

\section{Luis Arenas-Carmona}

Dept. de Matemáticas

Facultad de Ciencias, Universidad de Chile, Casilla 653, Santiago, Chile.

e-mail : learenas@u.uchile.cl 